\title{
QUASI-SUPRABARRELLED SPACES
}

\section{J. C. FERRANDO and M. LÓPEZ-PELLICER}

(Received 13 April 1987; revised 14 September 1987)

Communicated by J. H. Rubinstein

\begin{abstract}
In this paper a proper class of barrelled spaces which strictly contains the suprabarrelled spaces is considered. A closed graph theorem and some permanence properties are given. This allows us to prove the necessity of a condition of a theorem of S. A. Saxon and P. P. Narayanaswami by constructing an example of a non-suprabarrelled Baire-like space which is a dense subspace of a Fréchet space and is not an $(L F)$-space under any strong locally convex topology.
\end{abstract}

1980 Mathematics subject classification (Amer. Math. Soc.) (1985 Revision): primary 46 A 07; secondary 46 A 30.

Keywords and phroses: barrelled space.

In [9], S. A. Saxon and P. P. Narayanaswami prove that if $E$ is a Fréchet space and $F$ is a barrelled dense subspace, then $F$ is not suprabarrelled if and only if there exists a subspace $G$ of $E$ such that $G$ contains $F$ and $G$ with a topology stronger than the relative one is an $(L F)$-space. In this paper we give an example which allows us to prove that $G$ cannot be replaced by $F$ in the former theorem. We introduce the quasi-suprabarrelled spaces which satisfy the inclusion relationships indicated by

$$
\text { suprabarrelled } \Rightarrow \text { quasi-suprabarrelled } \Rightarrow \text { barrelled. }
$$

A closed graph theorem together with some permanence properties are given. We use some of those results later in order to construct the quoted example.

The linear spaces we use are defined over the field of the reals or complex numbers. By "space" we mean "Hausdorff locally convex space". If $A$ is a (C) 1989 Australian Mathematical Society 0263-6115/89 \$A2.00 + 0.00 
subset of a linear space we denote by $\langle A\rangle$ its linear span. If $B$ is a bounded closed absolutely convex subset of a space $E, E_{B}$ denotes the normed space over the linear hull of $B$. If $\left\{E_{i}: i \in I\right\}$ is a family of spaces, $E:=\prod\left\{E_{i}: i \in I\right\}$ and $J$ is a part of $I$, we write $E(J)$ to denote the subspace of $E$ of those elements which have zero in any coordinate position indexed by $I-J$. A space $E$ is suprabarrelled or $(d b),[12],[6]$ and [9], if given an increasing sequence of subspaces of $E$ covering $E$ there is one of them which is barrelled and dense in $E$. A space $E$ is Bairelike, [7], if given an increasing sequence of closed absolutely convex subsets of $E$ covering $E$ there is one of them which is a neighbourhood of the origin. A space $E$ is quasi-Baire, [7], if it is barrelled and given an increasing sequence of subspaces of $E$ covering $E$ there is one of them which is dense.

\section{Quasi-suprabarrelled spaces and the closed graph theorem}

We say that a space $E$ is quasi-suprabarrelled if given an increasing sequence of subspaces of $E$ covering $E$ there is one of them which is barrelled.

We have that suprabarrelled implies quasi-suprabarrelled and this last class of spaces is contained in the class of the barrelled ones. The following examples distinguish these classes.

EXAMPLE 1. A non-complete $(L F)$-space is not quasi-suprabarrelled. In fact, if $E(\tau)$ is quasi-suprabarrelled, from the sequence $\left\{E_{n}\left(\tau_{n}\right): n=1,2, \ldots\right\}$ of Fréchet spaces which defines $E(\tau)$ we can extract a sequence $\left\{E_{n(p)}\left(\tau / E_{n(p)}\right): p=\right.$ $1,2, \ldots\}$ of barrelled spaces which are of Fréchet by Pták's homomorphism theorem, since the identity $i: E_{n(p)}\left(\tau_{n(p)}\right) \rightarrow E_{n(p)}\left(\tau / E_{n(p)}\right)$ is continuous. Now $E(\tau)$ is the strict inductive limit of the $E_{n(p)}\left(\tau / E_{n(p)}\right)$ since $E(\tau)$ is barrelled, [7, Lemma 2.17]. We must conclude then that $E(\tau)$ is complete, [3, p. 225], a contradiction. We note that if besides $E(\tau)$ is metrizable, then $E(\tau)$ is Baire-like.

The L. Schwartz space $D(\Omega)$ is not quasi-suprabarrelled as we are going to show. We first note that every separated quotient of an $(L F)$-space is an $(L F)$ space or a Fréchet space $([2$, p. 147 , lines 5.6]; also, see [4, Theorem 9]). Actually, let $E(\tau)=\underset{n}{\lim _{n}} E_{n}\left(\tau_{n}\right)$ be an $(L F)$-space and let $H$ be a closed subspace of $E$. Every canonical mapping from $E_{n}\left(\tau_{n}\right) / H \cap E_{n}$ into $E(\tau) / H$ is continous. Therefore the identity $i$ from $\underset{n}{\lim } E_{n}(\tau) / H \cap E_{n}$ onto $E(\tau) / H$ is continuous. The conclusion follows now from Grothendieck's closed graph theorem. As $D(\Omega)$ has a noncomplete metrizable quotient $F,[14]$, and every quotient of a quasisuprabarrelled space is quasi-suprabarrelled as we shall see later, by the above $F$ is not quasi-suprabarrelled and thus neither is $D(\Omega)$. 
EXAMPLE 2. The topological direct sum $\phi \omega=\omega \oplus \omega \oplus \cdots$ is complete and barrelled. Now $\phi \omega$ is covered by the subspaces $R_{n}=\omega^{n} \times \phi_{\omega}$, where $\phi_{\omega}$ denotes $\phi$ with the topology induced by $\omega$. No $F_{n}$ is barrelled, since $\phi_{\omega}$ is not-barrelled, and so $\phi \omega$ is not quasi-suprabarrelled.

EXAMPLE 3 . The space $\phi$ is quasi-suprabarrelled since every subspace is of countable codimension, ([11] and [8]). Now $\phi$ is covered by an increasing sequence of finite-dimensional subspaces, therefore $\phi$ is not quasi-Baire and so $\phi$ is not suprabarrelled. In fact, if $d$ is a cardinal number and $\phi_{d}$ denotes a linear space of dimension $d$ with the strongest locally convex topology, then every subspace of $\phi_{d}$ has the strongest locally convex topology and, therefore, is closed and barrelled. It follows that $\phi_{d}$ is quasi-suprabarrelled. If $H$ is a countable codimensional subspace of $\phi_{d}$, then $\phi_{d}=H \oplus \phi$. As $\phi$ is not suprabarrelled we conclude that $\phi_{d}$ is not suprabarrelled.

We give now a characterization of quasi-suprabarrelled spaces which are not suprabarrelled.

PROPOSITION 1. Let $E$ be a quasi-suprabarrelled space. $E$ is not suprabarrelled if and only if there exists an increasing sequence of barrelled subspaces covering $E$ having each of them a copy of $\phi$ with a topological supplement.

PrOOF. Obviously, $E$ is suprabarrelled if and only if $E$ is quasi-Baire. Thus Theorem 1 of [4] applies.

It is obvious that a space $E$ is suprabarrelled if and only if it is quasisuprabarrelled and quasi-Baire. It follows from [4] that if $E$ does not contain a complemented copy of $\phi$ (in particular, if its completion is a Baire space (see [15]), then the notions of being quasi-suprabarrelled and suprabarrelled are equivalent for $E$.

Theorem 4 of [12] suggests the following result, where we change suprabarrelled by quasi-suprabarrelled, losing the localizatoin property given there. We suppose $E$ is the convex hull of a family of quasi-suprabarrelled spaces, that $\left\{F_{n}\left(\tau_{n}\right): n=1,2, \ldots\right\}$ is an increasing sequence of $\Gamma_{r}$-spaces, [13], and $F:=$ $\bigcup\left\{F_{n}: n=1,2, \ldots\right\}$ has a locally convex topology coarser than the final topology defined by the $F_{n}\left(\tau_{n}\right), n=1,2, \ldots$

THEOREM 1. If $f$ is a linear mapping from $E$ into $F$ with closed graph, then $f$ is continuous.

ProOF. We can suppose that $E$ is quasi-suprabarrelled. Working with a subsequence if necessary we can also suppose that every space $f^{-1}\left(F_{n}\right)$ is barrelled. From $f$ we consider the restrictions $f_{n}: f^{-1}\left(F_{n}\right) \rightarrow F_{n}\left(\tau_{n}\right)$ which clearly have closed graph in $f^{-1}\left(F_{n}\right) \times F_{n}\left(\tau_{n}\right)$. By the closed graph theorem given in [13] 
we have that the $f_{n}$ are continuous and from the continuity of $f_{n}: f^{-1}\left(F_{n}\right) \rightarrow F$ it follows that $f$ is continuous, since $E$ is the inductive limit of the subspaces $f^{-1}\left(F_{n}\right), n=1,2, \ldots$.

\section{Permanence properties of quasi-suprabarrelled spaces}

The quotients and the countable-codimensional subspaces of quasi-suprabarrelled spaces are quasi-suprabarrelled. This follows immediately from the analogous permanence properties of barrelled spaces.

PROPOSITION 2. Let $E_{1}$ and $E_{2}$ be two quasi-suprabarrelled spaces. If $E_{1}$ is metrizable, then $E_{1} \times E_{2}$ is quasi-suprabarrelled.

PROOF. If $E=E_{1} \times E_{2}$ is not quasi-suprabarrelled there exists an increasing sequence $\left\{F_{n}: n=1,2, \ldots\right\}$ of proper subspaces of $E$ covering $E$, none of them barrelled. Let $T_{n}$ be a barrel in $F_{n}$ which is not a neighbourhood of the origin in $F_{n}$. If $B_{p}$ denotes the closure of $T_{p}$ and $G_{p}:=\bigcap\left\{\left\langle B_{n}\right\rangle: n \geq p\right\}$, then $\left\{G_{p}: p=\right.$ $1,2, \ldots\}$ is an increasing sequence of subspaces of $E$ covering $E$, since $F_{p}$ is contained in $G_{p}$ for $p=1,2, \ldots$ No $G_{p}$ is barrelled, since $B_{p} \cap G_{p}$ is a barrel in $G_{p}$ which is not a neighbourhood of the origin in $G_{p}$.

Since $E_{1}$ and $E_{2}$ are quasi-suprabarrelled there is a strictly increasing sequence $(p(r))_{r}$ of positive integers such that $G_{p(r)} \cap E$, and $G_{p(r)} \cap E_{2}$ are barrelled for $r=1,2, \ldots$ By metrizability, $E_{1}$ is suprabarrelled and so there is a positive integer $q$ such that $G_{p(q)} \cap E_{1}$ is dense in $E_{1}$.

If $y \in E_{1}$ there is a sequence $\left(y_{n}\right)_{n}$ is the barrelled space $G_{p(q)} \cap E_{1}$ which converges to $y$. This sequence is absorbed by the barrels $B_{n} \cap G_{p(q)} \cap E_{1}$ for each $n \geq p(q)$. Hence $y \in\left\langle B_{n}\right\rangle$ for each $n \geq p(q)$. Thus $y \in G_{p(q)}$ and so we have proved that $E_{1}$ is contained in $G_{p(q)}$. Now, from this inclusion it follows that $G_{p(q)}=E_{1} \times\left(G_{p(q)} \cap E_{2}\right)$ is barrelled, a contradiction.

LEMMA 1. Let $\left\{E_{n}: n=1,2, \ldots\right\}$ be a sequence of closed absolutely convex sets in $E$. If $G_{p}:=\bigcap\left\{\left(B_{n}\right): n \geq p\right\}, p=1,2, \ldots$, and $(p(r))_{r}$ is a strinctly increasing sequence of positive integers with $\bigcup\left\{G_{p(r)}: r=1,2, \ldots\right\}=E$, there is a positive integer $q$ such that $G_{p(q)}$ contains $E(q, q+1, q+2, \ldots)$.

PROOF. If the property is not ture we find a sequence $\left(x_{r}\right)_{r}$ in $E$ with $x_{r} \in$ $E(r, r+1, \ldots)-G_{p(r)}$. This sequence converges to zero and is contained in a complete subspace of $E$ since its projection on $E_{n}$ is contained in a finite-dimensional subspace of $E_{n}$, therefore by Tychonoff's Theorem, $A=\overline{\Gamma\left\{x_{r}: r=1,2, \ldots\right\}}$ is a compact set and $E_{A}$ is a Banach space which is covered by the $G_{p(r)}, r=1,2, \ldots$ 
Thus, there exists a $G_{p(q)} \cap E_{A}$ which is of second category in $E_{A}$. Therefore $B_{n} \cap E_{A}$ is a neighbourhood of the origin in $E_{A}$ for each $n \geq p(q)$. So we have that $A$ is contained in $\left\langle B_{n}\right\rangle$ for each $n \geq p(q)$. This implies that $A \subset G_{p(q)}$, contradicting the fact that $x_{q} \notin G_{p(q)}$.

Proposition 3. Let $\left\{E_{n}: n=1,2, \ldots\right\}$ be a sequence of spaces such that $E(1,2, \ldots, n)$ is quasi-suprabarrelled for $n=1,2, \ldots$ Then $E=\prod\left\{E_{n}: n=\right.$ $1,2, \ldots\}$ is quasi-suprabarrelled.

PROOF. If $E$ is not quasi-suprabarrelled there exists an increasing sequence $\left\{F_{n}: n=1,2, \ldots\right)$ of proper subspaces of $E$ covering $E$ such that no $F_{n}$ is barrelled. If $T_{n}$ is a barrel in $F_{n}$ which is not a neighbourhood of the origin in $F_{n}, B_{p}$ is the closure of $T_{p}$ in $E$ and $G_{p}:=\bigcap\left\{\left\langle B_{n}\right\rangle: n \geq p\right\}$, we saw in the proof of Proposition 2 that $\left\{G_{p}: p=1,2, \ldots\right\}$ is an increasing sequence of non-barrelled subspaces of $E$ which covers $E$.

We determine a subsequence $\left\{G_{p(r)}, r=1,2, \ldots\right\}$ with $p(r)<p(r+1)$ for each $r$ such that $G_{p(r)} \cap E(1,2, \ldots, r)$ is barrelled. In fact, since $E_{1}=E(1)$ is quasi-suprabarrelled, there exists a $p(1)$ such that $G_{p(1)} \cap E(1)$ is barrelled. With $p(1), p(2), \ldots, p(s-1)$ determined, we have that $\left\{G_{n}, n=1,2, \ldots\right\}$ is an increasing sequence of spaces covering the quasi-suprabarrelled space $E(1,2, \ldots, s)$, therefore there is a $G_{p(s)}, p(s)>p(s-1)$, such that $G_{p(s)} \cap E(1,2, \ldots, s)$ is barrelled.

Since $\bigcup\left\{G_{p(r)}, r=1,1, \ldots\right\}=E$, by Lemma 1 there is a positive integer $q$ such that $G_{p(q)} \supset E(q, q+1, \ldots)$. Thus $G_{p(q)}$ is the topological direct sum of the subspaces $G_{p(q)} \cap E(1,2, \ldots, q)$ and $E(q+1, q+2, \ldots)$. Therefore $G_{p(q)}$, is barrelled, a contradiction.

EXAMPLE 4. We give now an example of a non-complete quasi-suprabarrelled space which is not suprabarrelled. In fact, there exists a linear form $u$ defined on $\phi^{N}$ which is not continuous. Setting $H:=u^{-1}(0), H$ is a dense hyperplane of $\phi^{N}$. As $\phi^{n}$ is isomorphic to $\phi$ for every $n \in N$, then it follows from the last result that $\phi^{N}$ is quasi-suprabarrelled. This implies that $H$ is quasi-suprabarrelled. If $H$ is suprabarrelled then its completion $\phi^{N}$ is suprabarrelled, [12], but $\phi^{N}$ fails to be suprabarrelled since it contains $\phi$ as a factor.

We need the following result given in $[15$, p. 20] by M. Valdivia.

LEMMA 2. Let $\left\{E_{i}: i \in I\right\}$ be a family of spaces and let $E=\prod\left\{E_{i}: i \in I\right)$. If $\left\{B_{n}: n=1,2, \ldots\right\}$ is a sequence of closed convex sets of $E$ covering $E$ such that $0 \in B_{n}$ for every positive integer $n$, there exists a finite subset $J$ of $I$ and $a$ positive integer $s$ such that $B_{s} \supset E(I-J)$. 
PROPOSITION 4. Let $\left\{E_{i}: i \in I\right\}$ be a family of spaces such that $E(H)$ is quasi-suprabarrelled for every countable set $H \subset I$. Then $E:=\prod\left\{E_{i}: i \in I\right\}$ is quasi-suprabarrelled.

PROOF. If $E$ is not quasi-suprabarrelled there exists an increasing sequence $\left\{F_{n}: n=1,2, \ldots\right\}$ of proper non-barrelled subspaces covering $E$. Let $T_{n}$ be a barrel in $F_{n}$ which is not a neighbourhood of the origin in $F_{n}$ and let $B_{n}$ be the closure of $T_{n}$ in $E$. Since $\left\{m B_{n}: m, n=1,2, \ldots\right\}$ covers $E$ it follows from Lemma 2 that there is a pair $(m(1), n(1))$ of positive integers and a finite subset $H_{1}$ of $I$ such that $m(1) B_{n(1)} \supset E\left(I-H_{1}\right)$. Thus $\left\langle B_{n(1)}\right\rangle \supset E\left(I-H_{1}\right)$. Now $\left.\left\{m B_{n}: n\right) n(1), m=1,2, \ldots\right\}$ covers $E$ and therefore there is a $n(2)>n(1)$ such that $\left\langle B_{n(2)}\right\rangle \supset E\left(I-H_{2}\right)$. By recurrence we obtain a sequence $n(1)<n(2)<$ $\cdots<n(p)<\cdots$ of positive integers and a sequence $\left\{H_{p}: p=1,2, \ldots\right\}$ of finite subsets of $I$ such that $\left\langle B_{n(p)}\right\rangle \supset E\left(I-H_{p}\right)$ for $p=1,2, \ldots$.

Let $H:=\bigcup\left\{H_{p}: p=1,2, \ldots\right\}$ and $G_{p}:=\bigcap\left\{\left\langle B_{n(r)}: r \geq p\right\}\right.$. Then $\left\{G_{p}: p=\right.$ $1,2, \ldots\}$ is an increasing sequence of subspaces of $E$ covering $E$ with $G_{p} \supset$ $E(I-H)$ for $p=1,2, \ldots$. Since $H$ is countable we have that $E(H)$ is quasisuprabarrelled, therefore there is a positive integer $q$ such that $G_{q} \cap E(H)$ is barrelled. Now $G_{q}$ is the topological direct sum of $G_{q} \cap E(H)$ and $E(I-H)$, so $G_{q}$ is barrelled, a contradiction.

THEOREM 2. Let $\left\{E_{i}: i \in I\right\}$ be a family of spaces such that for every finite subset $H$ of $I E(H)$ is quasi-suprabarrelled. Then $E=\prod\left\{E_{i}: i \in I\right\}$ is quasisuprabarrelled.

PROOF. It is a direct consequence of Propositions 3 and 4.

EXAMPLE 5. From Example 3 and from the last theorem it follows that $\prod\left\{\phi_{d}: d \in \Omega\right\}$, where $\Omega$ is an arbitrary family of cardinals numbers, is a quasisuprabarrelled space which is not suprabarrelled. In particular, if $I$ is an index set, $\phi^{I}$ is quasi-suprabarrelled and it is not suprabarrelled.

REMARK 1 A space $E$ satisfies condition $(G)$, [5], if given a sequence of subspaces of $E$ covering $E$ there is one of them which is barrelled. A space $E$ is said to be totally barrelled, [16], if given a sequence of subspaces of $E$ covering $E$ there is one of them which is Baire-like (see Proposition 1 of [5]). In [16] it is proved that $l_{0}^{\infty} \otimes_{\pi} l^{2}$ is a suprabarrelled space which is not totally barrelled. Now, it is known that if $E$ satisfies condition $(G)$ and $E$ does not contain a copy of $\phi$ then $E$ is totally barrelled. As $l_{0}^{\infty} \otimes_{\pi} l^{2}$ is metrizable, this space does not contain a copy of $\phi$. We must conclude then that $l_{0}^{\infty} \otimes_{\pi} l^{2}$ is a quasi-suprabarrelled space which does not satisfy condition $(G)$. 


\section{Proof of the necessity of a condition in a theorem of S. A. Saxon and P. P. Narayanaswami}

We consider in the product space $\omega^{N}$ the sequence $\left\{E_{n}, n=1,2, \ldots\right\}$ of nonbarrelled subspaces of the form $E_{n}=\omega \times .^{(n)} \cdot \times \omega \times \phi_{\omega} \times \phi_{\omega} \times \cdots$, where $\phi_{\omega}$ denotes $\phi$ with the topology induced by $\omega$, and the subspaces $E:=\bigcup\left\{E_{n}: n=\right.$ $1,2, \ldots\}$ and $F:=\phi_{\omega}^{N}$. Clearly the space $E$ is dense in $\omega^{N}$ and it is not quasisuprabarrelled since none of the spaces $E_{n}, n=1,2, \ldots$ is barrelled.

Let $E(\delta)$ be the barrelled space which is the inductive limit of the quasisuprabarrelled spaces $E_{n}\left(\delta_{n}\right)=\omega \times \ldots(n) \cdot \times \omega \times \phi \times \phi \times \cdots$. From the next result it follows that $E$ is barrelled.

\section{Proposition 5. E and $E(\delta)$ are isomorphic.}

PROOF. If $u \in E(\delta)^{\prime}$ we have that $u / E_{n} \in E_{n}\left(\delta_{n}\right)^{\prime}=\phi \oplus . .(n) \cdot \oplus \phi \oplus \omega \oplus \omega \oplus \cdots$. Analogously, $u / E_{n+1} \in \phi \oplus .{ }^{(n+1)} \cdot \oplus \phi \oplus \omega \oplus \omega \oplus \cdots$. Since $\left(u / E_{n+1}\right) / E_{n}=u / E_{n}$, it follows that $u / E_{n+1} \in \phi \oplus .{ }^{(n)} \cdot \oplus \phi \oplus \omega \oplus \omega \oplus \cdots$. Proceeding by recurrence we obtain that $u / E_{n}$ is an element of $\phi \oplus \phi \oplus \phi \oplus \cdots$ independent of $n$. This element defines a linear form on $\omega^{N}$ and, therefore, on $E$. Hence, $u \in E^{\prime}$. As $E^{\prime} \subset E(\delta)^{\prime}$, we conclude that $E$ and $E(\delta)$ are isomorphic since they are two Mackey spaces with the same dual.

PROPOSITION 6. If $\tau$ is a locally convex topology strictly stronger than that of $E$, then $E(\tau)$ is not an $(L F)$-space.

Proof. If $E(\tau)$ is an $(L F)$-space, then the identity $i: E \rightarrow E(\tau)$ is continuous as a consequence of the Theorem 1. Thus $\tau$ must coincide with the topology of E.

The next result can be viewed either as a special case of Lemma 2, due to $\mathrm{M}$. Valdiva, [15, page 20] or as a special case of A. Todd and S. Saxon's Lemma 4.5 of $[10]$. We provide a concise proof.

LEMMA 3 . If the product $L=\prod\left\{L_{n}: n=1,2, \ldots\right\}$ is covered by an increasing sequence $\left\{F_{n}: r=1,2, \ldots\right\}$ of closed subspaces, then there is a positive integer $m$ such that $F_{m} \supset\{0\} \times \cdots \times\{0\} \times L_{m+1} \times L_{m+2} \times \cdots$.

PROOF. If the property is not true, there is some $x_{n} \in\{0\} \times \cdots \times\{0\} \times L_{n+1} \times$ $L_{n+2} \times \cdots-F_{n}$ for every $n \in N$. Now the compact $A:=\overline{\Gamma\left\{x_{n}: n=1,2, \ldots\right\}}$ generates a Banach space $E_{A}$ which is covered by the sequence $\left\{F_{n} \cap E_{A}: n=\right.$ $1,2, \ldots\}$. One of these subspaces, $F_{P} \cap E_{A}$, is of second category in $E_{A}$ and, therefore, coincides with $E_{A}$ since it is closed in $E_{A}$. It follows that $A \subset F_{P}$. But $x_{P} \notin F_{P}$. Contradiction. 
PROPOSITION 7. $E$ is not an $(L F)$-space.

Proof. Suppose that $E$ is the inductive limit of the increasing sequence $\left\{F_{n}\left(\rho_{n}\right): n=1,2, \ldots\right\}$ of Fréchet spaces covering $E$.

Working with a subsequence if necessary we can suppose, since $F(\delta)=\phi^{N}$ is quasi-suprabarrelled (see Example 4), that $F \cap F_{n}$ is a barrelled subspace of $F(\delta)$, $n=1,2, \ldots$, which we are going to show is closed. Now the injections $i_{1}$ and $i_{2}$ from $F \cap F_{n}$ and $F_{n}\left(\rho_{n}\right)$ into $\omega^{N}$ respectively, are continuous. So the injection $i$ from $F \cap F_{n}$ into $F_{n}\left(\rho_{n}\right)$ has closed graph, hence it is continuous. If $\left\{z_{i}: i \in I, \geq\right\}$ is a net in $F \cap F_{n}$ which converges to $z$ in $F(\delta)$ we have from the continuity of $i$ that $\left\{z_{i}: i \in I, \geq\right\}$ converges to $v$ in $F_{n}\left(\rho_{n}\right)$ and from the continuity of $i_{1}$ and $i_{2}$ that $z=v$. Therefore $\left\{F \cap F_{n}: n=1,2, \ldots\right\}$ is an increasing sequence of closed subspaces covering $\phi^{N}$. The previous lemma implies that there is a positive integer $m$ such that $F \cap F_{m} \supset\{0\} \times .^{m} \cdot \times\{0\} \times \phi \times \phi \times \cdots$.

The Fréchet space $F_{m}\left(\rho_{m}\right)$ is covered by $\left\{E_{n} \cap F_{m}: n \geq m\right\}$, hence there exists an $s \geq m$ such that $E_{s} \cap F_{m}$ is a Baire subspace dense in $F_{m}\left(\rho_{m}\right)$. The injections $j_{1}$ and $j_{2}$ from $E_{s} \cap F_{m}$ and $E_{s}\left(\delta_{s}\right)$ into $\omega^{N}$ respectively, are continuous. So the injection $j$ from $E_{s} \cap F_{m}$ into $E_{s}\left(\delta_{s}\right)$ is continuous since $j$ has a closed graph, $E_{s} \cap F_{m}$ is a Baire space and $E_{s}\left(\delta_{s}\right)$ is a webbed space. We prove just as before that $E_{s} \cap F_{m}$ is a closed subspace of $F_{m}\left(\rho_{m}\right)$. And from density we conclude that $E_{s} \supset F_{m}$.

Setting $H_{s}:=\{0\} \times \cdot{ }^{(s)} \cdot \times\{0\} \times \phi \times \phi \times \cdots$, we have that $H_{s} \subset F_{m} \subset E_{s}$. Now, endowing $H_{s}$ with the topology induced by $E_{s}\left(\delta_{s}\right)$ it follows that the injections $k$ and $l$ from $H_{s}$ into $F_{m}\left(\rho_{m}\right)$ and from $F_{m}\left(\rho_{m}\right)$ into $E_{s}\left(\delta_{s}\right)$ respectively, are continuous since they have closed graph, $H_{s}$ is barrelled, $F_{m}\left(\rho_{m}\right)$ is a Fréchet space and $E_{s}\left(\delta_{s}\right)$ is a webbed space. We must conclude that the topology of the non-metrizable space $H_{s}$ coincides with the topology induced by the Fréchet space $F_{m}\left(\rho_{m}\right)$. Contradiction.

In brief, the space $E$ has the following properties: (1) $E$ is dense in $\omega$. (2) $E$ is Baire-like. (3) $E$ is not quasi-suprabarrelled. (4) $E(\tau)$ is not an $(L F)$-space under any locally convex topology $\tau$ stronger that the topology induced by $\omega$. (5) $E$ is an inductive limit of a sequence of quasi-suprabarrelled spaces. (6) $E$ is a webbed space.

REMARK 2. If $s$ is the subspace of $\omega$ of the rapidly decreasing sequences, $s$ is a Fréchet space under the topology defined by the sequence of seminorms of the form $\|x\|_{P}=\sum\left\{n^{P}\left|x_{n}\right|: n=1,2, \ldots\right\}, p=0,1,2, \ldots$ In [17] it is proved that if $G_{n}:=\omega \times \cdots(n) \cdot \times \omega \times s \times s \times \cdots$ and $G:=\left\{G_{n}: n=1,2, \ldots\right\}$, then $G$ is a proper dense subspace of $\omega$ which is an $(L F)$-space. Our space $E$ is a subspace of this space $G$. Having in mind this fact it also follows from the quoted theorem of S. A. Saxon and P. P. Narayanaswami that $E$ is not suprabarrelled. 


\section{References}

[1] M. DeWilde, Closed graph theorems and webbed spaces (Pitman, London, San Francisco, Melbourne, 1978).

[2] A. Grothendieck, Topological vector spaces (Gordon and Breach, New York, London, Paris, 1973).

[3] G. Köthe, Topological vector spaces I (Springer-Verlag, Berlin, Heidelberg, New York, 1969).

[4] P. P. Narayanaswami and S. A. Saxon, ' $(L F)$-spaces, quasi-Baire spaces and the strongest locally convex topology', Math. Ann. 274 (1986), 627-641.

[5] P. Pérez and J. Bonet, 'Remarks and examples concerning suprabarrelled and totally barrelled spaces', Arch. Math. 39 (1982), 340-347.

[6] W. Robertson, I. Tweddle and F. Yeomans, 'On the stability of barrelled topologies III', Bull. Austral. Math. Soc. 22 (1980), 99-112.

[7] S. A. Saxon, 'Nuclear and product spaces, Baire-like spaces and the strongest locally convex topology', Math. Ann. 197 (1972), 87-106.

[8] S. A. Saxon and M. Levin, 'Every countable-codimensional subspace of a barrelled space is barrelled', Proc. Amer. Math. Soc. 29 (1971), 91-96.

[9] S. A. Saxon and P. P. Narayanaswami, 'Metrizable ( $L F)$-spaces, (db)-spaces and the separable quotient problem', Bull. Austral. Math. Soc. 23 (1981), 65-80.

[10] A. Todd and S. A. Saxon, 'A property of locally convex Baire spaces', Math. Ann. 206 (1973), 23-34.

[11] M. Valdivia, 'A bsolutely convex sets in barrelled spaces', Ann. Inst. Fourier 21 (1971), 3-13.

[12] M. Valdivia, 'On suprabarrelled spaces', Functional Anal., Holomorphy and Approximation Theory, Rio de Janeiro 1978, pp. 572-580, Lecture Notes in Mathematics 843, Springer, Berlin, Heidelberg, New York, (1981).

[13] M. Valdivia, Śobre el teorema de la gráfica cerrada', Collect. Math. 22 (1971), 51-72.

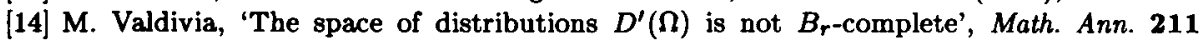
(1974), 145-149.

(15] M. Valdivia, Topics in locally convex spaces (Math. Studies, North-Holland, Amsterdam, New York, Oxford, 1982).

[16] M. Valdivia and P. Pérez Carreras, 'On totally barrelled spaces', Math. Z. 1978 (1981), 263-269.

[17] M. Valdivia and P. Pérez Carreras, 'Sobre espacios $(L F)$ metrizables', Collect. Math. 33 (1982), 297-303.

Departamento de Matemáticas (ETSIA)

Universidad Politécnica

Apartado 22012

46022-Valencia

Spain 